\title{
Tuberculosis case finding using population- based disease surveillance platforms in urban and rural Kenya
}

Godfrey Bigogo ${ }^{1 *}$, Kevin Cain², Diana Nyole', Geoffrey Masyongo', Joshua Auko Auko', Newton Wamola', Albert Okumu', Janet Agaya', Joel Montgomery², Martien Borgdorff ${ }^{2,3}$ and Deron Burton ${ }^{2}$

\begin{abstract}
Background: Tuberculosis (TB) case finding is an important component of TB control because it can reduce transmission of Mycobacterium tuberculosis (MTB) through prompt detection and treatment of infectious patients.

Methods: Using population-based infectious disease surveillance (PBIDS) platforms with links to health facilities in Kenya we implemented intensified TB case finding in the community and at the health facilities, as an adjunct to routine passive case finding conducted by the national TB program. From 2011 to 2014, PBIDS participants $\geq 15$ years were screened either at home or health facilities for possible TB symptoms which included cough, fever, night sweats or weight loss in the preceding 2 weeks. At home, participants with possible TB symptoms had expectorated sputum collected. At the clinic, HIV-infected participants with possible TB symptoms were invited to produce sputum. Those without HIV but with symptoms lasting 7 days including the visit day had chest radiographs performed, and had sputum collected if the radiographs were abnormal. Sputum samples were tested for the presence of MTB using the Xpert MTB/RIF assay. TB detection rates were calculated per 100,000 persons screened.

Results: Of 11,191 participants aged $\geq 15$ years screened at home at both sites, 2695 (23.9\%) reported possible TB symptoms, of whom 2258 (83.8\%) produced sputum specimens. MTB was detected in $32(1.4 \%)$ of the specimens resulting in a detection rate of 286/100,000 persons screened. At the health facilities, a total of 11,762 person were screened, 7500 (63.8\%) had possible TB symptoms of whom $1282(17.1 \%)$ produced sputum samples. MTB was detected in 69 (5.4\%) of the samples, resulting in an overall detection rate of 587/100,000 persons screened. The TB detection rate was higher in persons with HIV compared to those without at both home (HIV-infected - 769/100,000, HIV-uninfected 141/100,000, rate ratio (RR) - 5.45, 95\% Cl 3.25-22.37), and health facilities (HIV-infected 3399/100,000, HIV-uninfected 294/100,000, RR 11.56, 95\% Cl 6.18-18.44).

Conclusion: Facility-based intensified TB case finding detected more TB cases per the number of specimens tested and the number of persons screened, including those with HIV, than home-based TB screening and should be further evaluated to determine its potential programmatic impact.
\end{abstract}

Keywords: Tuberculosis, Case-finding, Kenya

\footnotetext{
* Correspondence: Gbigogo@kemricdc.org

${ }^{1}$ Centre for Global Health Research, Kenya Medical Research Institute, P.O Box

1578 -, Kisumu 40100, Kenya

Full list of author information is available at the end of the article
}

(c) The Author(s). 2018 Open Access This article is distributed under the terms of the Creative Commons Attribution 4.0 International License (http://creativecommons.org/licenses/by/4.0/), which permits unrestricted use, distribution, and

reproduction in any medium, provided you give appropriate credit to the original author(s) and the source, provide a link to the Creative Commons license, and indicate if changes were made. The Creative Commons Public Domain Dedication waiver (http://creativecommons.org/publicdomain/zero/1.0/) applies to the data made available in this article, unless otherwise stated. 


\section{Background}

A central aim of tuberculosis (TB) control programs is to quickly identify new TB cases and ensure that these cases successfully complete treatment [1-3]. Prompt treatment of persons with TB disease halts their infectiousness and reduces transmission of $\mathrm{TB}$, resulting in a decline in TB incidence in the community [4]. Case detection is an important component in TB programs, but it can be influenced by several factors including health care access, care seeking practices and diagnostic capabilities [5-7]. TB programs in many countries rely primarily on passive TB case finding where patients self-refer to clinics and are screened for TB based on the attending clinician's judgment. However, in many parts of the developing world with poor access to health facilities, passive case finding alone may not achieve the global target of $70 \%$ case detection rate $[1,8,9]$. In sub-Saharan Africa, TB diagnosis and treatment have not been prompt with delays ranging from 50 to 180 days reported $[5,6,10-12]$.

Early detection and treatment are important in reducing transmission of the tuberculosis bacilli [13-15]. Intensified case finding among household members of infectious TB cases is an effective approach to curb TB spread $[16,17]$. However in areas with high rates of TB and HIV, important sources of infection may be contacts outside the household, thus a broader, community-based approach combined with enhanced screening at health facilities may be needed to improve TB case finding in such settings $[9,14,15,18-23]$. We examined the yield of intensified TB case finding at community level and in health facilities among persons $\geq 15$ years of age as an adjunct to the passive facility based case detection method in areas with high TB and HIV prevalence in urban and rural Kenya.

\section{Methods}

\section{Study sites}

The Kenya Medical Research Institute (KEMRI), in collaboration with the Centers for Disease Control and Prevention (CDC) have conducted population-based infectious disease surveillance (PBIDS) in two sites in Kenya since 2005; in Kibera, an urban slum in Nairobi, and in Lwak in rural western Kenya (Fig. 1) [24, 25]. The two study sites have referral facilities - Lwak hospital in the rural site and Tabitha clinic in the urban site where participants can access free care for all potentially infectious disease syndromes, and can access TB clinics that operate 5 days a week (Monday - Friday). Besides the TB clinics at the referral facilities, there were several

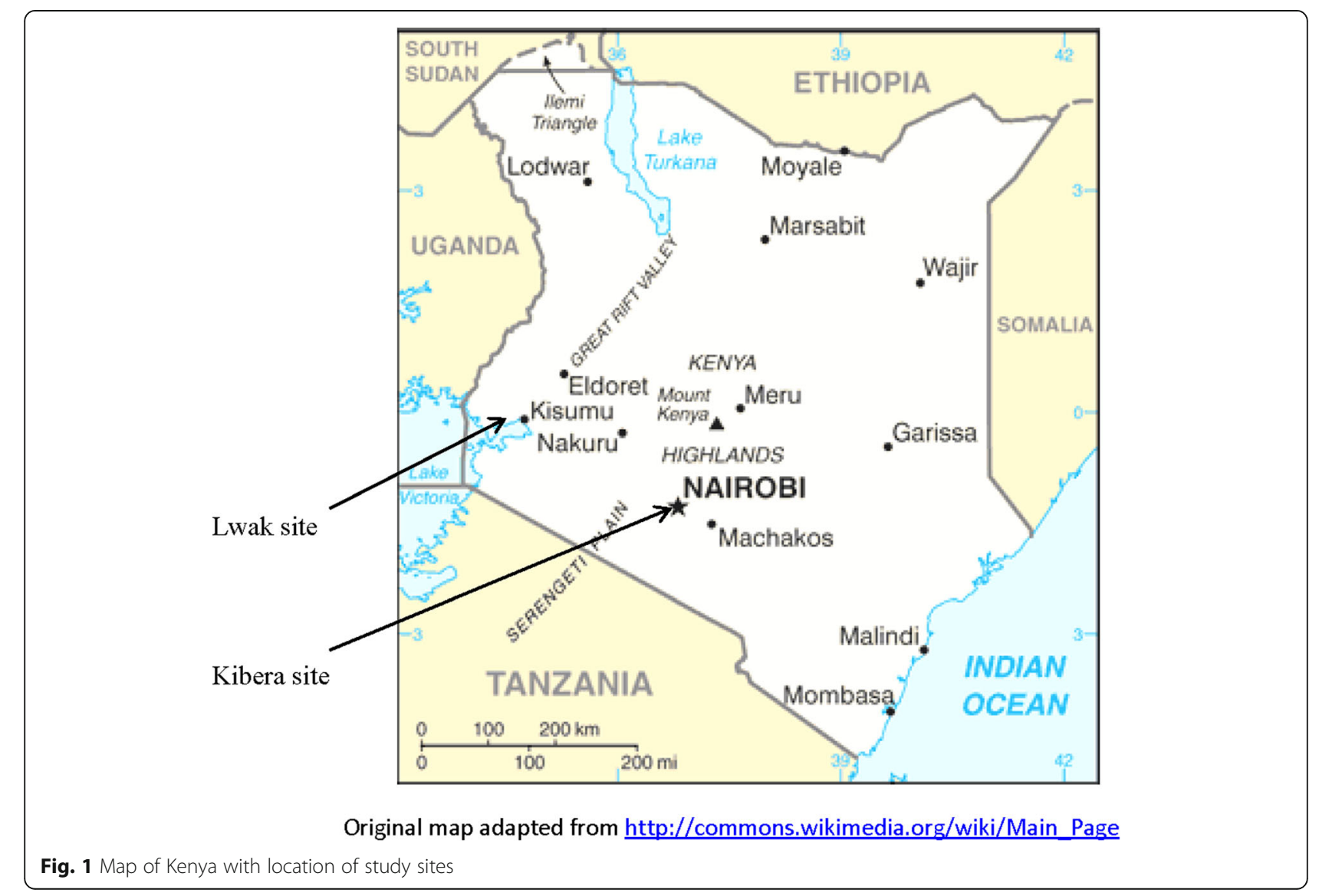


other TB clinics run by the Ministry of Health within the PBIDS areas. Some PBIDS participants visited these clinics for care.

\section{Kibera site}

The surveillance area located in Kibera is $<0.5 \mathrm{~km}^{2}$, and has a population between 25,000 and 29,000 since PBIDS began; population density is 77,000 people per $\mathrm{km}^{2}$. Nearly all (98\%) residents in the area under surveillance are enrolled in PBIDS [24]. Residents live in houses built of mud or brick walls with tin roofs. Open sewers flow along footpaths and water is mostly obtained from vendors. Most individuals living in the slum work as casual laborers or engage in small businesses. HIV prevalence in the area in the years 2009-2010 was $15 \%$ among individuals aged $\geq 18$ years [26]. Prior to the implementation of intensified TB case finding, baseline data abstracted from TB registers from health facilities in the study area indicated an annual TB notification rate for persons $\geq 15$ years of 567 cases per 100,000 persons between 2009 and 2011 (unpublished PBIDS data). Other than Tabitha clinic, there are two additional clinics within the study area that offered TB screening (through sputum microscopy) of patients based on clinicians' assessments.

\section{Lwak site}

The Lwak site is situated in Siaya County in rural western Kenya. The study site and the socio-economic characteristics of the population in this area have been described previously $[25,27,28]$. Briefly, the PBIDS catchment population approximated at $24,000-26,000$ is drawn from individuals living in 33 villages within a $5 \mathrm{~km}$ radius of Lwak hospital, a private hospital that serves as the project's referral facility. The population density is 320 people per $\mathrm{km}^{2}$. Besides Lwak hospital, there are three government-run health facilities which conduct passive TB case finding in the area. A TB prevalence study done in the area in the years 2006-2008 found a prevalence of 600 cases per 100,000 persons [29]. Baseline PBIDS data estimated a TB notification rate of 706 cases per 100,000 persons for the years 2009-2011. HIV prevalence in the years 2008-2009 was estimated at $17 \%$ among adults [26].

\section{Data collection and analysis}

\section{Household TB screening and specimen collection}

As part of ongoing disease surveillance since 2005, participants in the PBIDS project were visited weekly or bi-weekly at home by field workers who used standardized questionnaires to gather information about any illnesses since the last home visit [25, 30-32]. At the onset, the study focused on characterization of four syndromes, which were pneumonia, diarrhea febrile illness and jaundice. Data on illnesses and care seeking for reported illnesses were recorded in programmed personal digital assistants (PDAs). Specific symptoms of illness asked for included cough, fever, difficulty in breathing, diarrhea, and yellow eyes. Abbreviated physical exams including axillary temperature measurements were carried out on ill persons present during the visit. Characterization of TB was not part of the original scope of the PBIDS surveillance project, and positive responses to the original routine symptom questions were not used to make referrals for TB testing. Rather, positive responses to the original symptom questions were used to make referrals for further evaluation of the four syndromes mentioned above.

From August to December 2011 for the Lwak site and from January to April 2012 for the Kibera site, active home screening for TB was conducted in which additional symptoms, including presence of weight loss and night sweats, were collected. Participants aged $\geq 15$ years who reported any cough, fever or night sweats in the previous 2 weeks, or weight loss over the month prior to the interview date were considered to have possible TB. They were consented and requested to produce sputum specimens in private inside their houses, or in the open outside of their houses. They were taught how to make a "huff cough" to help produce sputum. Field workers stored the sputum samples in boxes with ice packs which were maintained at $+2-+8{ }^{\circ} \mathrm{C}$. Sample collectors picked up the samples from the field workers and ferried them to a central location at Lwak hospital or Tabitha clinic, where the samples were registered in logs, batched and stored in refrigerators at $+2-+8{ }^{\circ} \mathrm{C}$ awaiting shipment to KEMRI laboratories in Kisumu or Nairobi.

\section{Facility-based TB screening and specimen collection}

PBIDS participants aged $\geq 15$ years old who presented to Tabitha clinic (from April 2012 to March 2014) or Lwak hospital (from May 2012 to April 2014) were asked if they had any of the following four possible TB symptoms: cough, fever, night sweats or weight loss in the last 1 month. Questions about the latter two symptoms were added as part of the TB case-finding intervention. Any participant reporting any of these symptoms was evaluated further according to a set algorithm (Fig. 2). In brief, HIV-infected participants with at least one of these symptoms were invited to produce sputum. Participants without HIV but with possible TB symptoms lasting at least 7 days including the day of the sick visit had a chest radiograph performed and were asked for sputum if the radiograph was abnormal. Sputum samples were logged and stored in refrigerators at $+2-+8{ }^{\circ} \mathrm{C}$ until shipment to the study laboratory. 


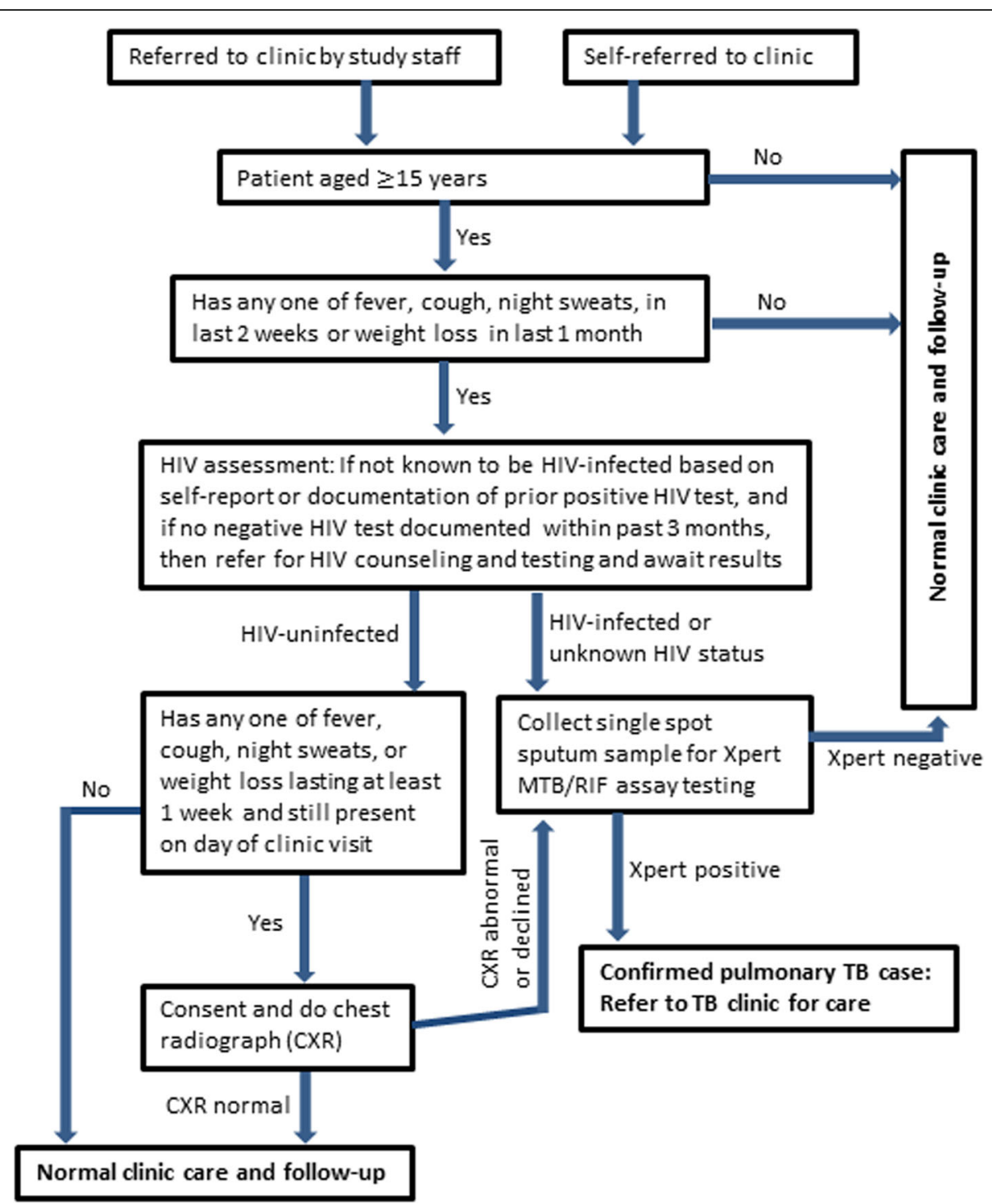

Fig. 2 Facility based TB screening flowchart used at the PBIDS health facilities in Lwak and Kibera

\section{Laboratory testing and follow-up}

Sputum samples were delivered daily from the study clinics to the KEMRI laboratories in Kisumu or Nairobi. Testing of the samples was done by Xpert MTB/RIF assay, which uses molecular techniques to detect the presence of Mycobacterium tuberculosis (MTB) and determine whether the bacterium is resistant to rifampicin [33, 34]. Test results were available after $2 \mathrm{~h}$. A confirmed pulmonary TB case was defined by a single positive Xpert result. Smear microscopy was performed on specimens from confirmed pulmonary TB cases in accordance with national TB program policy in Kenya [35].

All febrile patients had malaria tests and a subset had blood cultures done as described elsewhere [25]. Those with severe acute respiratory illness had blood cultures done [36].
Laboratory results were linked to participants' clinical data collected at home or at the study facilities. All TB results (positive and negative) were disseminated back to the participants by field workers within 2 weeks. Participants with confirmed pulmonary TB were invited to come to the study facilities and were referred to the onsite TB clinics, or were asked to visit a TB clinic of their choice for further evaluation and care according to Kenya's national TB program policy [35]. All positive TB test results were shared with the Kenyan Ministry of Health, Division of TB and Leprosy. Participants with negative TB test results but who still experienced possible TB symptoms 1 month after initial $\mathrm{TB}$ testing were invited to be retested. 


\section{HIV status data}

Large-scale home based HIV counseling and testing (HBCT) was performed in the years 2008-2009 when all participants $\geq 13$ years in the PBIDS areas were offered HIV testing as described previously [26, 37]. Seventy-eight percent of eligible adults $\geq 18$ years and $87 \%$ of youth aged 13-17 years agreed to be tested during that period [26]. In subsequent years, HIV testing targeted adult new PBIDS participants and previously HIV-uninfected residents who requested to be re-tested. For those participants not retested after the home-based HIV counseling and testing campaign, we assumed that their HIV status at the time of home-based testing was the same throughout the study period. This assumption is supported by the low estimated annual HIV transmission in the study areas $(<1 \%$ based on KEMRI unpublished data).

HIV counseling and testing were offered routinely at the study referral facilities for all participants aged $\geq 15$ years. For this project, previous HIV positive results were accepted. Participants with previous HIV negative results $>3$ months old or those with an unknown HIV status were invited to be tested for HIV infection during the period of intensified TB case finding.

\section{Data analysis}

We describe demographic and clinical characteristics of PBIDS participants with confirmed pulmonary $\mathrm{TB}$ at the two study sites. TB detection rates were calculated as the number of cases per 100,000 persons screened, disaggregated by age group, gender, and HIV status. We used Poisson regression to calculate rate ratios and compare TB detection rates by age, gender and HIV status among persons screened at home and at study health facilities. We controlled for clustering of cases at household level using general estimating equations. For this analysis we assumed an individual's HIV status at home screening was the same as that established at HBCT unless there were different results from more recent tests. Analyses were done using STATA 13.1 Software (StataCorp, Texas USA).

\section{Ethical consideration}

The protocol was reviewed and approved by the Kenya Medical research Institute (KEMRI) Ethics Review Committee (No. 1899) and the Centres for Disease Control and Prevention (CDC) Institutional Review Board (No. 4566). All participants provided consent to participate in the study. Participants aged 15-17 years provided assent after parental consent was obtained.

\section{Results}

Screening for possible TB symptoms and results of TB sputum testing

At home, 6575/13,740 (47.9\%) and 4616/12,864 (35.9\%) of PBIDS participants aged $\geq 15$ years in Lwak and
Kibera, respectively, were available for TB symptom screening. Of the total 11,191 PBIDS participants screened at home, $2695(24.1 \%)$ reported possible TB symptoms of whom 2258 (83.8\%) had sputum successfully collected. Of the participants with sputum available for testing, 32 (1.4\%) participants (28 at initial round and 4 at retesting 1 month later) were confirmed to have pulmonary TB. TB positivity of sputum specimens collected at home was similar across the two sites; $1.2 \%$ $(18 / 1546)$ in Lwak (Table 1), and 2.0\% (14/712) in Kibera (Fisher's exact $p$-value $=0.178$ ).

At the study health facilities 5765/13,800 (41.8\%) and $5997 / 11,448(52.4 \%)$ of the resident populations were screened in Lwak and Kibera, respectively. Of the total 11,762 participants screened at the two health facilities, $7500(63.8 \%)$ were found with possible TB symptoms, of whom $1282(17.1 \%)$ had sputum collected. TB was detected in $69(5.4 \%)$ of the sputum samples obtained at the health facilities. TB positivity of sputum specimens was $4.6 \%(28 / 607)$ at Lwak hospital (Table 1), and 6.1\% $(41 / 675)$ at Tabitha clinic in Kibera (Fisher's exact $p$-value $=0.266)$.

\section{Demographic and clinical characteristics of participants with confirmed pulmonary TB}

Of the 101 participants confirmed to have pulmonary TB across both study sites (32 from home-based screening, 69 from facility-based screening), 51 (50\%) were males and 55 (54\%) were HIV-infected (Table 2). The mean age of participants with confirmed TB was 37 years (range 19-77 years). Cough was the most common symptom reported in $75(74 \%)$ of the confirmed pulmonary TB cases. Overall, 49.5\% (50/101) of participants with confirmed pulmonary $\mathrm{TB}$ had positive sputum smear microscopy, ranging from 35.7\% (10/28) among those screened for TB at Lwak hospital to $57.1 \%(8 / 14)$ among those screened at home in Kibera. Among participants with confirmed pulmonary $\mathrm{TB}$ at the health facilities who had blood cultures (13 total) or blood smears for malaria (17 total) done, two patients in each category were co-infected (15\% positive blood cultures; $12 \%$ positive malaria smear). All participants with confirmed pulmonary TB (101/101) were referred to TB clinics for care and the Ministry of Health was notified of their TB test results.

\section{Detection rate of confirmed pulmonary TB among screened participants according to study site, screening location, and individual characteristics}

The detection rate of confirmed pulmonary TB among persons screened in person at home was 274/100,000 persons (95\% CI 162-432/100,000 persons) in Lwak and $303 / 100,000$ persons (95\% CI $164-508 / 100,000$ persons) in Kibera (Table 1). At the health facilities, the TB 


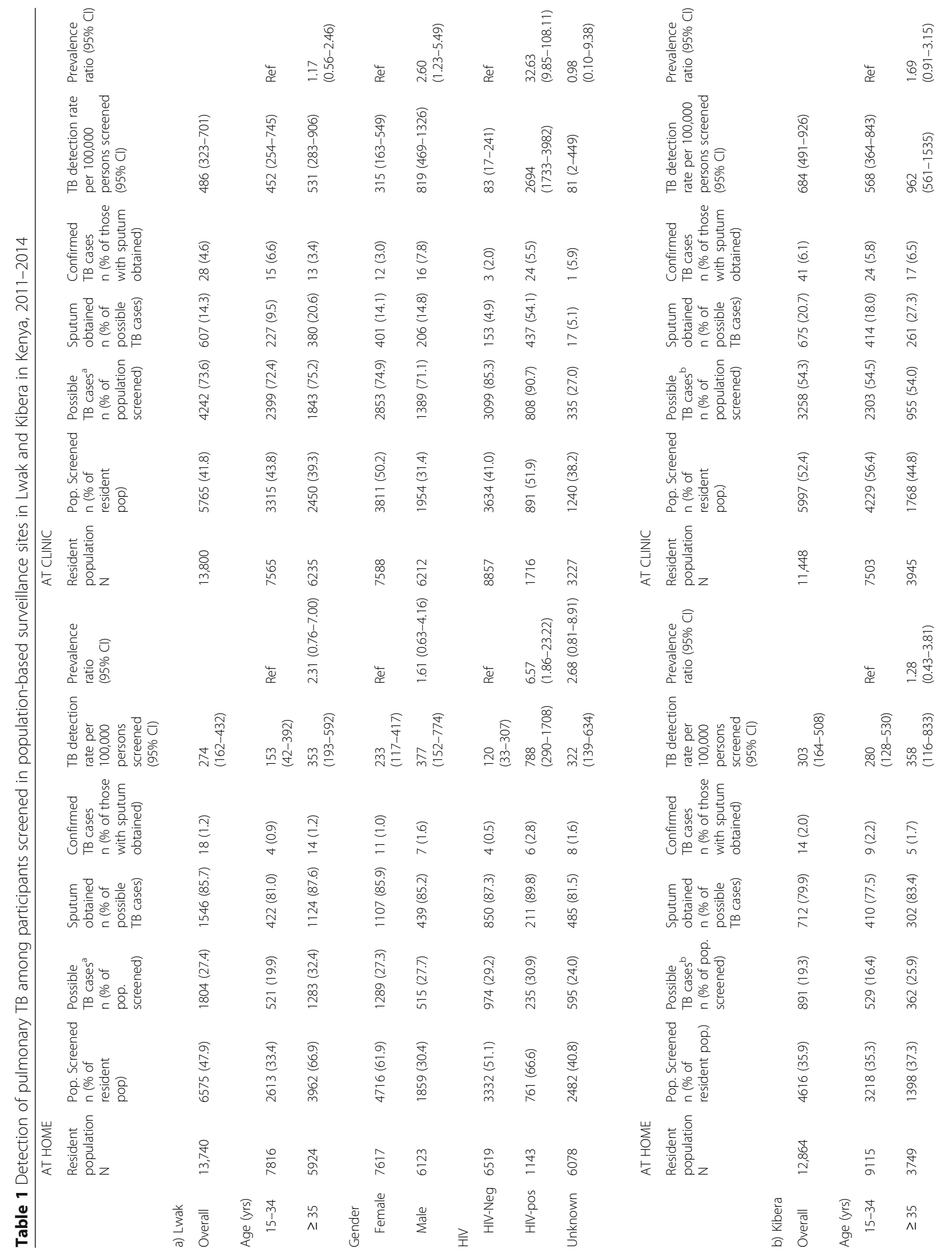




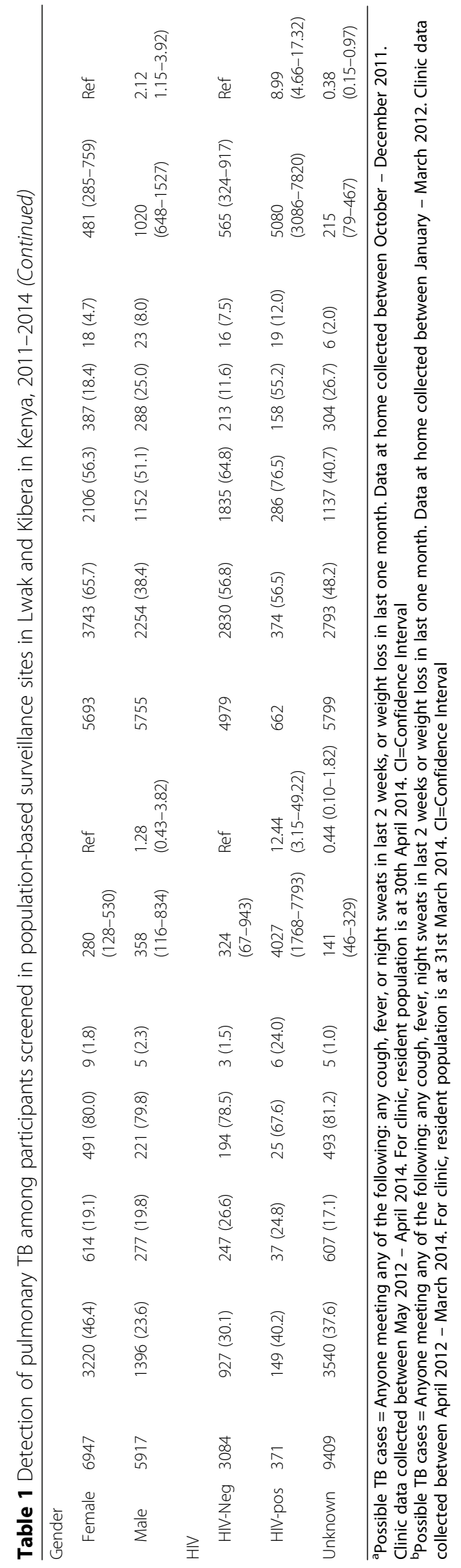


Table 2 Characteristics of participants $\geq 15$ years with confirmed pulmonary TB in Lwak and Kibera study sites in Kenya, 2011-2014

\begin{tabular}{|c|c|c|c|c|c|}
\hline \multirow[t]{4}{*}{$\underline{\text { Lwak }}$} & \multirow{4}{*}{$\begin{array}{l}\text { From home } \\
\text { screening } \\
\mathrm{N}=18 \\
\mathrm{n}(\%)\end{array}$} & \multirow{4}{*}{$\begin{array}{l}\text { From facility-based } \\
\text { screening }^{\text {b }} \\
N=28 \\
\mathrm{n}(\%)\end{array}$} & \multicolumn{3}{|l|}{ Kibera } \\
\hline & & & $\begin{array}{l}\text { From home } \\
\text { screening }\end{array}$ & $\begin{array}{l}\text { From facility-based } \\
\text { screening }\end{array}$ & Total \\
\hline & & & $N=14$ & $N=41$ & $N=101$ \\
\hline & & & $n(\%)$ & $n(\%)$ & $n(\%)$ \\
\hline Mean age & 51 & 41 & 34 & 33 & 37 \\
\hline (Min - max) & $(23-77)$ & $(21-56)$ & $(24-54)$ & $(19-59)$ & 19-77 \\
\hline \multicolumn{6}{|l|}{ Gender } \\
\hline Male & $7(38.9)$ & $16(57.1)$ & $5(35.7)$ & $23(56.1)$ & $51(50.5)$ \\
\hline \multicolumn{6}{|l|}{ HIV status } \\
\hline Positive & $6(33.3)$ & $24(85.7)$ & $6(42.9)$ & $19(46.3)$ & $55(54.5)$ \\
\hline Negative & $4(22.2)$ & $3(10.7)$ & $3(21.4)$ & $16(39.0)$ & $26(25.7)$ \\
\hline Unknown & $8(44.4)$ & $1(3.6)$ & $5(35.7)$ & $6(14.6)$ & $20(19.8)$ \\
\hline \multicolumn{6}{|l|}{ Socioeconomic status $^{c}$} \\
\hline Low & $9(50.0)$ & $5(17.9)$ & - & - & $\begin{array}{l}14 / 46 \\
(30.4)\end{array}$ \\
\hline Middle & $4(22.2)$ & $16(57.1)$ & - & - & $\begin{array}{l}20 / 46 \\
(43.5)\end{array}$ \\
\hline High & $5(27.8)$ & $6(21.4)$ & - & - & $\begin{array}{l}11 / 46 \\
(23.9)\end{array}$ \\
\hline Not available & $0(0)$ & $1(3.6)$ & - & - & $1 / 46(2.2)$ \\
\hline Previous history of $\mathrm{TB}^{\mathrm{d}}$ & $4(22.2)$ & - & $3(21.4)$ & - & $7 / 32(21.9)$ \\
\hline \multicolumn{6}{|l|}{ Symptoms reported } \\
\hline Cough & $16(88.9)$ & $20(71.4)$ & $9(64.3)$ & $30(73.2)$ & $75(74.3)$ \\
\hline Fever & $3(16.7)$ & $15(53.6)$ & $6(42.9)$ & $6(14.6)$ & $30(29.7)$ \\
\hline Night sweats & $6(33.3)$ & $8(28.6)$ & $5(35.7)$ & $5(12.2)$ & $24(23.8)$ \\
\hline Weight loss & $4(22.2)$ & $7(25.0)$ & $5(35.7)$ & $5(12.2)$ & $21(20.8)$ \\
\hline \multicolumn{6}{|l|}{ Duration of symptoms ${ }^{e}$} \\
\hline$\leq 2$ weeks & $14(77.8)$ & - & $8(57.1)$ & - & $22(21.8)$ \\
\hline$>2$ weeks & $4(22.2)$ & - & $6(42.9)$ & - & $10(9.9)$ \\
\hline \multicolumn{6}{|l|}{ Coinfections $^{f}$} \\
\hline Positive blood culture & - & 2/13 (15.4) & - & - & 2/13 (15.4) \\
\hline $\begin{array}{l}\text { Positive malaria } \\
\text { smear }\end{array}$ & - & $1 / 16(6.3)$ & - & $1 / 1(100)$ & 2/17 (11.8) \\
\hline Smear positive TB & $9(50)$ & $10(35.7)$ & $8(57.1)$ & $23(56.1)$ & $50(49.5)$ \\
\hline MoH Notified & $18(100)$ & $28(100)$ & $14(100)$ & $41(100)$ & $101(100)$ \\
\hline Referred to TB clinic & $18(100)$ & $28(100)$ & $14(100)$ & $41(100)$ & $101(100)$ \\
\hline
\end{tabular}

aTB screening at home occurred in October - December 2011 for Lwak, and January - March 2012 for Kibera, and the column contains only individuals screened in-person

${ }^{b}$ Facility-based TB screened occurred between May 2012 - April 2014 for Lwak, and April 2012 - March 2014 for Kibera. Individuals were referred from home or could self-refer to the health facilities

'Socioeconomic status classes computed based on asset ownership were available for Lwak site only

$\mathrm{d}, \mathrm{e}$ Data available from home only

fData available for health facilities only. Denominators are the number of persons who had either a blood culture or a malaria test done among those who had a positive TB result

detection rate in Lwak hospital was 486/100,000 persons (95\% CI 323-701/100,000 persons), and in Tabitha clinic was $684 / 100,000$ persons ( $95 \%$ CI $491-926 / 100,000$ persons) (Table 1). Overall, across both study sites, the TB detection rate was significantly higher for screening performed at the referral facilities compared to homes (referral facility TB detection rate: 587/100,000 persons, 95\% CI 457-742/100,000 persons; home-based TB detection rate: 286/100,000 persons, 95\% CI 196-403/ 100,000 persons; rate ratio (RR) $2.05,95 \%$ CI $1.35-3.12$ ). 
At the health facilities, TB detection rates were approximately 2 -fold higher in men than women (RR 2.60, 95\% CI 1.23-5.49 in Lwak hospital; RR 2.12, 95\% CI 1.15-3.92 in Tabitha clinic) (Table 1). The TB detection rate did not differ by gender for home-based TB screening at either site. For both facility-based and home-based screening, at both sites, there was a trend towards higher $\mathrm{TB}$ detection rates among persons aged $\geq 35$ years compared to those aged 15-34 years, though these trends were not statistically significant (Table 1).

In home-based screening at Lwak, the TB detection rate among persons infected with HIV was 6-fold higher compared to those without HIV; 788/100,000 (95\% CI 290-1708/100,000) among HIV-infected persons compared to $120 / 100,000 \quad(95 \%$ CI 33-307) among HIV-uninfected persons, RR 6.57 (95\% CI 1.86-23.22). The detection rate of 4027/100,000 (95\% CI 1768-7793/ 100,000) among HIV-infected persons screened at home in Kibera was 12-fold higher compared to the rate of 324/100,000 (95\% CI 67-943) among HIV-uninfected persons; RR 12.44, (95\% CI, 3.15-49.22) (Table 1).

In facility-based TB screening across study sites, TB detection rates among persons infected with HIV were 9and 33-fold higher compared to those without HIV in Kibera and Lwak respectively (Table 1). In Lwak the rate was 2694/100,000 (95\% CI 1733-3982/100,000) persons among HIV-infected persons compared to $83 / 100,000$ (95\% CI 17-241/100,000) persons, among HIV-uninfected; RR 32.63 (95\% CI 9.85-108.11). In Kibera the detection rate was $5080 / 100,000$ persons (95\% CI 3086-7820/100,000 persons) among HIV-infected persons compared to $565 / 100,000$ (95\% CI 324-917/100,000), among HIV-uninfected; RR 8.97 (95\% CI, 4.66-17.32).

\section{Discussion}

We implemented community- and health facility-based active TB case-finding using existing population-based infectious disease surveillance platforms in rural and urban sites in Kenya. In both sites we observed greater TB detection rates among participants screened for TB at health facilities compared to those screened at home. Our study was unique in applying similar TB case finding strategies in well-established rural and urban PBIDS sites with distinct demographic and epidemiologic profiles using electronic data collection systems, as adjuncts to the Ministry of Health's passive TB case detection methods. The detailed characterization of study participants within the PBIDS platforms enabled us to calculate screening coverage and TB detection rates stratified by various demographic and clinical characteristics, and the close proximity of referral health facilities and KEMRI laboratories facilitated rapid $\mathrm{TB}$ diagnosis using the Xpert MTB/RIF assay, which at the time of the study was not widely available in many areas in Kenya.
At both study sites, overall TB detection rates and the proportion of sputum specimens that were positive for TB were higher in participants screened at health facilities compared to those screened at home, suggesting that intensified TB case finding at health facilities may be a more efficient approach than home-based TB screening. Nevertheless, in settings with low health care seeking, home-based or other forms of community-based TB active case-finding might be important complementary approaches to facility-based TB case-finding, particularly for high-risk populations such as persons living with HIV, who had high TB detection rates at home in both of our study sites (788 and 4027 per 100,000 persons screened in the rural and urban site, respectively).

Community-based case finding strategies also have the potential to identify persons with TB early in the course of their disease and link them to treatment $[9,22]$, which may produce better treatment outcomes and reduce community transmission. In both our rural and urban study sites, the majority of participants diagnosed with TB through home-based intensified case finding reported symptoms that began $\leq 2$ weeks prior to screening. Although it is not known how long these participants identified at home with confirmed pulmonary TB would have remained undetected through routine passive, facility-based $\mathrm{TB}$ case finding, the observation that $50-57 \%$ of TB cases identified at home were smear positive suggests that there is a strong possibility that these participants might have contributed to additional TB transmission in the community before seeking care. Given that delays in diagnosis have been identified as one of the obstacles in the control of TB $[6,11]$, intensified home-based TB case finding may be an important tool in TB control in appropriate settings and should be further evaluated to determine its long-term impact on TB incidence through its utility in early diagnosis of $\mathrm{TB}$.

We found very high TB detection rates among persons living with HIV, both at home (1319, 95\% CI 683-2292 per 100,000 persons) and at the referral health facilities (3399, 95\% CI 2471-4552 per 100,000 persons). In sub-Saharan Africa, there is a virulent synergy between HIV and TB [16, 17, 22]. Of the 9.6 million incident cases of TB in 2014, an estimated 1.15 million (12\%) were HIV positive; $74 \%$ of these HIV-positive cases were in the African region [3]. Our rural and urban PBIDS sites have a high prevalence of HIV estimated at $15 \%$ for persons $\geq 13$ years [26]. In similar settings, it may be particularly beneficial to implement multifaceted intensified TB case finding among persons living with HIV, including both community-based and health facility-based strategies. Notably, uptake of sputum collection was significantly increased among persons living with HIV screened at home $(87 \%)$ versus screened at the health 
facilities (55\%). It is unclear whether this difference reflected operational issues around sputum collection at the health facilities, or increased participant acceptance of sputum collection in more private home settings, or other factors, but it is possible that $\mathrm{TB}$ detection rates from intensified case finding at the health facilities would have been substantially increased if a greater proportion of eligible HIV-infected participants had provided sputum samples. Effective targeting of intensified TB case finding to persons living with HIV requires robust HIV counseling and testing services with high community uptake. Strategies such as home based counseling and testing for HIV have been deployed in several African countries and have proved successful in identifying HIV infected persons [38, 39]. TB case finding efforts at community level may be aligned with HBCT efforts to increase success in identifying persons co-infected with TB and HIV.

Among participants without HIV, the TB detection rate in the urban site was greater than in the rural site for both home-based and facility-based intensified case finding. Several studies have documented high TB burden in slum settings. In a study conducted in a densely populated informal settlement in Dhaka, Bangladesh, the prevalence of TB was four-fold higher than that recorded in other urban areas with less dense populations [40]. Similarly, in an informal settlement in Site-M in Cape Town in South Africa, the TB incidence was significantly higher in densely populated areas than less dense regions [41]. Kibera in Nairobi, where our urban PBIDS site is located, is one of the largest slums in Africa and is 240-times more densely populated than our rural PBIDS site in western Kenya. It is likely that crowded living conditions increases $\mathrm{TB}$ transmission in the urban site.

Our home-based intensified TB case finding strategy was designed to be an adjunct to the existing passive, facility-based case finding system used by the national TB program in Kenya. While the home-based case-finding strategy was successful in quickly identifying several TB cases over a short span of time at both rural and urban sites and linking them to treatment, the overall yield from this strategy was low relative to what might have been expected based on earlier estimates of TB prevalence in the study sites [29]. The reasons for the relatively low numbers of $\mathrm{TB}$ cases identified at home are not clear, but it is likely that we missed some participants who were symptomatic and eligible for TB testing but were absent from home at the time of the TB screening visits. For instance, most of the participants screened at both home and the health facilities were women, but the TB detection rate was consistently increased among men, particularly at the health facilities where the $\mathrm{TB}$ detection rate was 2-fold greater compared to women. In some settings, home-based intensified TB case finding strategies may need to be supplemented with central community-level TB screening methods (such as mobile testing units) with extended hours to increase uptake among potentially high-risk sub-populations that are less likely to be found at home during the day [29].

Another potential limitation of our home-based screening method is that possible TB symptoms that were not present at the time of the home visit may have been underreported because of limited recall, as has been demonstrated previously [27]. Additionally, it is possible that some participants with $\mathrm{TB}$ but who were asymptomatic in the 2 weeks prior to the home visit were missed, as the symptom screening only collected information about symptoms experienced within the past 2 weeks. Lastly, both at home and the health facilities, sputum production was challenging for some participants with possible TB symptoms, particularly those without cough. Availability of induced sputum services at the referral facilities might have increased our TB detection yield.

The yield of our facility-based TB active case finding may have been impacted by the preceding home-based TB case finding interventions that occurred in each PBIDS site. We would expect that after an intensive community TB screening activity, there would be fewer undiagnosed TB cases seen at the health facilities. However, in both study sites, the yield of the facility-based $\mathrm{TB}$ case finding was greater than that of the home-based case finding in terms of both counts and detection rates. Partly, this may reflect that substantial proportions of the PBIDS populations (52 and 64\% in Lwak and Kibera, respectively) were not screened at home during the community $\mathrm{TB}$ active case finding activities, leaving a number of potential TB cases in the communities undetected. Nevertheless, it may be that the facility-based intensified TB case finding interventions would have had even greater yield if they had not followed rounds of active home-based TB screening.

We also are unable to ascertain whether our TB active case finding activities resulted in additional TB case notifications beyond what would have occurred without these interventions. As noted above, TB cases identified through our study interventions may eventually have been detected through routine, passive facility-based $\mathrm{TB}$ case finding. In this circumstance, one might expect that our interventions would produce a temporary bolus of increased TB case notifications (based on earlier detection), followed potentially by a period of decreased case notifications during the timeframe when the TB cases that we diagnosed would otherwise have been detected through passive means. TB case notifications also might have declined in the time period following the study 
interventions because of reduced TB transmission associated with earlier treatment of infectious TB cases identified during the study than otherwise would have occurred. Alternatively, our intensified TB case finding activities might have resulted in a true overall increase in TB case notifications, if some of the TB cases that we identified would never have been detected otherwise. We do not have information available to determine which scenario has occurred. There are no published TB case notification data that are fully overlapping with the PBIDS populations that we could use to assess changes in notifications over time for our study participants. However, as a future step, we intend to explore the possibility of abstracting data from TB registers at clinics in and around the study sites, and linking those data by name and demographic factors to PBIDS data to identify study participants, to examine trends in TB case notifications in our study populations in the periods preceding, during, and following our intensified $\mathrm{TB}$ case finding interventions.

\section{Conclusion}

In summary, we evaluated intensified $\mathrm{TB}$ case finding strategies implemented at home and in referral health facilities among persons $\geq 15$ years of age in established rural and urban PBIDS sites in Kenya, as an adjunct to the Ministry of Health's routine, passive facility-based TB case detection methods. Facility-based intensified TB case finding yielded greater TB detection rates than home-based screening, but both case finding strategies identified high rates of TB among persons living with HIV. Both strategies should be evaluated further for their potential programmatic impact on TB control in appropriate settings.

\section{Abbreviations}

CDC: US Centers for disease control and prevention; Cl: Confidence interval; HBCT: Home based counseling and testing; HIV: Human immune-deficiency virus; KEMRI: Kenya medical research institute; MTB: Mycobacterium tuberculosis; PBIDS: Population based infectious disease surveillance; PDA: Personal digital assistant; RIF: Rifampicin; RR: Rate ratio; TB: Tuberculosis

\section{Acknowledgements}

We would like to thank PBIDS participants in Lwak and Kibera sites for agreeing to be part of this study. Secondly, although at times faced with harsh weather, the field teams in the two sites endeavored to complete their work as planned and deserve praise. The ministry of health teams at the sites collaborated well with study teams in initiating follow-ups of confirmed TB patients. We are grateful for their collaboration with our study team. Lastly we would like to thank our staff in the lab notably Mr. Ben Muchiri, for conducting specimen analyses, and Mr. Allan Audi for data management efforts. This work has been cleared for publication by the Director, KEMRI.

\section{Funding}

The study was funded by a grant from the US Centers for Disease Control and Prevention through a cooperative agreement with KEMRI.

\section{Availability of data and materials}

Some of the variables used in the analysis may be made available on reasonable request to the corresponding author. Appropriate approvals will be needed from relevant organs within KEMRI before release of data.

\section{Disclaimer}

The findings and conclusions in this report are those of the author(s) and do not necessarily represent the official position of the Centers for Disease Control and Prevention.

\section{Authors' contributions}

GB; Conceived project, provided overall oversight for field and lab work at the two sites, analyzed data and wrote manuscript. KC; contributed to project implementation plan, reviewed manuscript. DN; coordinated fieldwork activities in Lwak, oversaw specimen collection, contributed to preliminary analysis of data, reviewed manuscript. GM; coordinated fieldwork activities in Kibera, oversaw specimen collection, reviewed manuscript. JAA; Collation of data from both sites, assisted in analyses, reviewed manuscript. NW; oversaw testing of specimens from Kibera site, preparation of lab results and reviewed manuscript. $\mathrm{AO}$; oversaw testing of specimens from Lwak site, preparation of lab results and reviewed manuscript. JA; technical assistance in fieldwork, review of data and manuscript. JM; Provided technical assistance in field and laboratory aspects, reviewed data and manuscript. MB: Contributed to conception of the project, provided technical assistance in fieldwork, reviewed data and manuscript. DB; contributed to conception and overall oversight of project, participated in collection and review of data, provided critical edits and review of manuscript. All authors have read and approved the contents of this manuscript and agreed to its publication.

\section{Ethics approval and consent to participate}

The protocol was reviewed and approved by the Kenya Medical research Institute (KEMRI) Ethics Review Committee (SSC 1899) and the Centres for Disease Control and Prevention (CDC) Institutional Review Board (\#4566). Participants provided written consent to participate in the study. Participants aged 15-17 years provided assent after parental consent was obtained.

\section{Competing interests}

The authors declare that they have no competing interests.

\section{Publisher's Note}

Springer Nature remains neutral with regard to jurisdictional claims in published maps and institutional affiliations.

\section{Author details}

${ }^{1}$ Centre for Global Health Research, Kenya Medical Research Institute, P.O Box 1578 -, Kisumu 40100, Kenya. ${ }^{2}$ U.S. Centers for Disease Control and Prevention, Atlanta, USA. ${ }^{3}$ Academic Medical Centre, University of Amsterdam, Amsterdam, Netherlands.

Received: 31 October 2017 Accepted: 28 May 2018

Published online: 07 June 2018

References

1. WHO, The Global Plan To Stop TB 2011-2015. 2011.

2. Onozaki I, Raviglione M. Stopping tuberculosis in the 21st century: goals and strategies. Respirology. 2010;15(1):32-43.

3. WHO, Global Tuberculosis Report 2015.

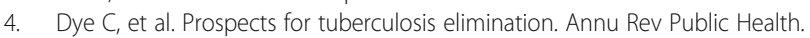
2013:34:271-86.

5. Jurcev-Savicevic A, et al. Health system delay in pulmonary tuberculosis treatment in a country with an intermediate burden of tuberculosis: a crosssectional study. BMC Public Health. 2013;13:250.

6. Storla DG, Yimer S, Bjune GA. A systematic review of delay in the diagnosis and treatment of tuberculosis. BMC Public Health. 2008;8:15.

7. Van't Hoog AH, et al. Risk factors for inadequate TB case finding in Rural Western Kenya: a comparison of actively and passively identified TB patients. PLoS One. 2013:8(4):e61162.

8. Abebe M, Doherty M, Wassie L, Demissies A, Mihret A, Engers H, et al. TB case detection: can we remain passive while the process is active? Pan Afr Med J. 2012;11:50

9. Eang MT, et al. Early detection of tuberculosis through community-based active case finding in Cambodia. BMC Public Health. 2012;12:469.

10. Ayisi JG, et al. Care seeking and attitudes towards treatment compliance by newly enrolled tuberculosis patients in the district treatment programme in rural western Kenya: a qualitative study. BMC Public Health. 2011;11:515. 
11. Ayuo PO, et al. Causes of delay in diagnosis of pulmonary tuberculosis in patients attending a referral hospital in Western Kenya. East Afr Med J. 2008; 85(6):263-8.

12. Wysocki $A D$, et al. Delay in seeking initial care for tuberculosis diagnosis. Rev Esc Enferm USP. 2013;47(2):440-7.

13. Chen $W$, et al. Pulmonary tuberculosis incidence and risk factors in rural areas of China: a cohort study. PLoS One. 2013;8(3):e58171.

14. Kompala T, Shenoi SV, Friedland G. Transmission of tuberculosis in resourcelimited settings. Curr HIV/AIDS Rep. 2013;10(3):264-72.

15. Wood R, et al. Tuberculosis transmission to young children in a South African community: modeling household and community infection risks. Clin Infect Dis. 2010;51(4):401-8.

16. Elden $\mathrm{S}$, et al. Integrating intensified case finding of tuberculosis into HIV care: an evaluation from rural Swaziland. BMC Health Serv Res. 2011;11:118.

17. Kranzer K, et al. Yield of HIV-associated tuberculosis during intensified case finding in resource-limited settings: a systematic review and meta-analysis. Lancet Infect Dis. 2010;10(2):93-102.

18. Becerra MC, et al. Expanding tuberculosis case detection by screening household contacts. Public Health Rep. 2005;120(3):271-7.

19. Claassens $M$, et al. High prevalence of tuberculosis and insufficient case detection in two communities in the Western Cape, South Africa. PLoS One. 2013;8(4):e58689.

20. Corbett EL, et al. Comparison of two active case-finding strategies for community-based diagnosis of symptomatic smear-positive tuberculosis and control of infectious tuberculosis in Harare, Zimbabwe (DETECTB): a cluster-randomised trial. Lancet. 2010;376(9748):1244-53.

21. Dowdy DW, et al. Population-level impact of active tuberculosis case finding in an Asian megacity. PLoS One. 2013;8(10):e77517.

22. Shapiro $A E$, et al. Community-based targeted case finding for tuberculosis and HIV in household contacts of patients with tuberculosis in South Africa. Am J Respir Crit Care Med. 2012;185(10):1110-6.

23. van't Hoog AH, et al. Screening strategies for tuberculosis prevalence surveys: the value of chest radiography and symptoms. PLoS One. 2012;7(7): e38691.

24. Breiman RF, et al. Population-based incidence of typhoid fever in an urban informal settlement and a rural area in Kenya: implications for typhoid vaccine use in Africa. PLoS One. 2012;7(1):e29119.

25. Feikin DR, et al. The burden of common infectious disease syndromes at the clinic and household level from population-based surveillance in rural and urban Kenya. PLoS One. 2011;6(1):e16085.

26. Dalal W, et al. Home-based HIV testing and counseling in rural and urban Kenyan communities. J Acquir Immune Defic Syndr. 2013:62(2):e47-54.

27. Feikin DR, et al. Evaluation of the optimal recall period for disease symptoms in home-based morbidity surveillance in rural and urban Kenya. Int J Epidemiol. 2010;39(2):450-8.

28. Odhiambo FO, et al. Profile: the KEMRI/CDC health and demographic surveillance system-Western Kenya. Int J Epidemiol. 2012;41(4):977-87

29. van't Hoog AH, et al. High prevalence of pulmonary tuberculosis and inadequate case finding in rural western Kenya. Am J Respir Crit Care Med. 2011;183(9):1245-53.

30. Bigogo GM, et al. Epidemiology of respiratory syncytial virus infection in rural and urban Kenya. J Infect Dis. 2013;208(Suppl 3):S207-16.

31. Otieno GA, et al. Caretakers' perception towards using zinc to treat childhood diarrhoea in rural western Kenya. J Health Popul Nutr. 2013;31(3):321-9.

32. Breiman RF, et al. Use of population-based surveillance to determine the incidence of rotavirus gastroenteritis in an urban slum and a rural setting in Kenya. Pediatr Infect Dis J. 2014;33(Suppl 1):S54-61.

33. Dorman SE, et al. Performance characteristics of the Cepheid Xpert MTB/RIF test in a tuberculosis prevalence survey. PLoS One. 2012;7(8):e43307.

34. Scott LE, et al. Comparison of Xpert MTB/RIF with other nucleic acid technologies for diagnosing pulmonary tuberculosis in a high HIV prevalence setting: a prospective study. PLoS Med. 2011;8(7):e1001061.

35. DLTLD, M.o.H., Kenya, DLTLD Strategic Plan (2011 - 2015). 2010.

36. Feikin DR, et al. Etiology and incidence of viral and bacterial acute respiratory illness among older children and adults in Rural Western Kenya, 2007-2010. PLoS One. 2012;7(8):e43656.

37. Bigogo $\mathrm{G}$, et al. The impact of home-based HIV counseling and testing on care-seeking and incidence of common infectious disease syndromes in rural western Kenya. BMC Infect Dis. 2014;14:376.
38. Granich RM, et al. Universal voluntary HIV testing with immediate antiretroviral therapy as a strategy for elimination of HIV transmission: a mathematical model. Lancet. 2009;373(9657):48-57.

39. Wachira J, et al. What is the impact of home-based HIV counseling and testing on the clinical status of newly enrolled adults in a large HIV care program in Western Kenya? Clin Infect Dis. 2012;54(2):275-81.

40. Banu $\mathrm{S}$, et al. Epidemiology of tuberculosis in an urban slum of Dhaka City, Bangladesh. PLoS One. 2013:8(10):e77721.

41. Bekker LG, Wood R. The changing natural history of tuberculosis and HIV coinfection in an urban area of hyperendemicity. Clin Infect Dis. 2010; 50(Suppl 3):S208-14.

\section{Ready to submit your research? Choose BMC and benefit from:}

- fast, convenient online submission

- thorough peer review by experienced researchers in your field

- rapid publication on acceptance

- support for research data, including large and complex data types

- gold Open Access which fosters wider collaboration and increased citations

- maximum visibility for your research: over $100 \mathrm{M}$ website views per year

At BMC, research is always in progress.

Learn more biomedcentral.com/submissions 